\title{
Research on the novel hardware configuration methods of the contact R-test based on the constraint models
}

\author{
Yongbing Zhou Guofu Ding Yuexinkai Zhang Lei Jiang* \\ (School of Mechanical Engineering, Southwest Jiaotong University, Chengdu, 610031, PR China)
}

Abstract: R-test is widely used to measure the rotary axis error of five-axis machine tools due to its high accuracy and convenient. There are some deficiencies in the research on measurement performance optimization such as the customized design under certain requirements. The novel hardware configuration methods of the contact R-test are proposed in this paper to realize customization. Firstly, the theoretical measurement model and the calibration model are established to be used as the measurement accuracy evaluation model. Secondly, the influence of hardware parameters on the measurement performance indexes of the measurement system is analyzed and the corresponding constraint models for measurement performance are established. Thirdly, the optimal configuration methods of hardware parameters based on constraint models are proposed using exhaustive search method and variable parameter method respectively. Finally, a prototype that is configured with the hardware parameters based on the above configuration methods, is developed to calibrate on the Coordinate Measuring Machine(CMM) and complete the measurement performance evaluation. The evaluation results show that the hardware configuration methods meet the certain measurement requirements without range and precision waste. The proposed methods provide guidance and reference for the customized design of contact R-test.

Keywords: Contact R-test; Customized Design; Hardware Configuration; Performance Evaluation

\section{Introduction}

Five-axis $\mathrm{CNC}$ machine tools have been widely used in the processing of complex parts in some fields such as automobiles and aerospace due to the advantages of high cutting efficiency and flexible tool position adjustment. However, the introduction of two rotating shafts complicates the mechanical structure and control system of the five-axis machine tool, and additional rotation error items are added. At this stage, there is no unified standard and specification for the measuring method of the axis of rotation error. Although measuring tools such as Double ball-bar(DBB $)^{[1,2]}$, laser tracker ${ }^{[3-5]}$ and laser interferometer ${ }^{[6]}$ can be used for identification and compensation, they all have certain limitations. In recent years, a high-efficiency and high-precision rotary axis error measuring device R-test has been developed. Similar to the DBB, the error measurement of the machine tool is achieved by the relative displacement of the master ball installed on the spindle and the three sensors installed on the base, and the R-test can obtain the three-directional relative displacement error.

Weikert firstly proposed the concept of the R-test, and proved its applicability in error

* Lei Jiang is corresponding author: jianglei0506@163.com 
measurement of five-axis $\mathrm{CNC}$ machine tools through a series of simulation experiments ${ }^{[7]}$. And then the R-test has attracted more and more attention from academia and industry. On the one hand, R-test is used to identify the positioning error of the rotating axis based on the ISO 230-7 static test rules at first ${ }^{[8]}$, and then the error detection method based on R-test is rated as one of the international standards for the positioning accuracy detection of five-axis CNC machine tools 错误: 找到引用源。, $[10]$. On the other hand, there are also related commercial products on the market, such as IBS in the Netherlands ${ }^{[11,12]}$ and FIDIA in Italy ${ }^{[13]}$.

R-test is divided into non-contact and contact according to different structures. The measuring accuracy of the non-contact R-test is not affected by contact wear and it has high safety during dynamic measurement, but it has very high requirements on materials and measuring environment due to the influence of the sensor's structure and detection performance, requiring regular maintenance and calibration, and the solution algorithm is complex. The 3D probe of the contact R-test adopts three contact displacement sensors, the displacement of the master ball can be directly expressed by the output of the sensors. The more important thing is the contact displacement sensor has the characteristics of high reliability, low cost and simple operation.

Currently, the research of R-test mainly includes five-axis CNC machine tool error measurement using the R-test directly, structural parameter optimization and part of the function extension, shown as following:

\section{Optimization of structural parameters}

Liu et al. obtained a spherical contact R-test device structure optimization design method based on the two indexes of maximum measuring space and measurement sensitivity, and search for the optimal value of these two key dimensions to complete structural optimization through Monte Carlo Method ${ }^{[14]}$. Li et al. derived the transformation model between the sensor reading and the three-direction displacement of the measuring sphere, and determined the main error factors affecting the error measurement device and analyzed the overall uncertainty of the device based on the mode ${ }^{[15]}$. Jiang et al. improved the calculation accuracy and efficiency of the spherical center coordinates using the adaptive differential evolution algorithm, optimized the structural parameters based on the measurement performance indicators, and finally formed system optimization methods to balance the measurement accuracy and $\cos ^{[16]}$.

(2) Static error measurement

Ibaraki S et al. developed the corresponding software, which can graphically present the test trajectory measured to the R-test to help users more intuitively understand the error motion of the rotating axis. And realized in the $\mathrm{CNC}$ system Error compensation parameter through digitizing the geometric error of the rotating axis ${ }^{[17]}$. Li et al. designed a simple and unified measurement method based on RTCP motion, and verifies it in the $\mathrm{A} / \mathrm{C}$ rotation axis error measurement process. By minimizing the number of conditions of the recognition matrix, the recognition accuracy is increased by $97 \%$, and finally the measurement efficiency of the rotary head is improved when the R-test detection system is used with auxiliary fixtures ${ }^{[18]}$. 
Zhong et al. proposed an S-shaped trajectory measuring method through scaling the machining path of the S-shaped test piece to the measuring range of the R-test to evaluate the dynamic accuracy of a five-axis machine tool, the dynamic accuracy of the five-axis machine tool can be reflected simply ${ }^{[19]}$. Brecher et al. proposed a dynamic R-test method for measuring the thermoelastic deviation of a five-axis machine tool under normal workshop environment temperature changes, the uncertainty of the measuring results and the correlation between the measuring error and the temperature were given ${ }^{[20]}$.

(4) Measuring function expansion

Hong et al. developed a non-contact R-test detection device based on the laser displacement sensor, to demonstrate that the two R-test detection devices are more conducive to revealing the error characteristics of the shaft compared to $\mathrm{DBB}^{[21]}$. Bitar-Nehme E. et al. used the thermal volume effect of the reference length under the axis cycle to quantify the influence of the activity of each axis on the Cartesian components of volume distortion based on the invar R-test device $\operatorname{and}^{[22]}$. Weng et al. developed a new type of R-test measurement system in consideration of the limitation of traditional measuring methods of machine tool thermal error due to specific processing conditions to achieve accurate thermal volume error measurement ${ }^{[23]}$. Guo et al. proposed a new type of non-contact R-test calibration scheme that reduced the positioning error of the calibration point and verification point by more than $80 \%$, and verified the effectiveness of the method in an industrial robot error measuring experiment ${ }^{[24]}$.

Users pay more and more attention to the cost control of measuring instrument with the increasing demand of R-test in engineering applications for error measurement of rotating mechanisms such as machine tools and robots. Therefore, it is necessary to achieve cost control of measuring instrument by customized design for the lower cost contact R-test. However, there are still some problems that need to be further studied and resolved:

(1) The hardware configuration of the R-test determines its measuring accuracy and range, and affects cost fluctuations at the same time. However, there is currently a lack of research on the hardware configuration of R-test for different performance requirements.

(2) The corresponding quantitative relationship between the performance indexes of the measurement system and the hardware parameters is not clear.

Therefore, this paper takes the flat contact R-test as the research object, proposes the hardware configuration methods to realize customization for the R-test, and the related methods can be easily extended to the contact R-test with other sensors. The main contribution points are as follows:

(1) The theoretical measurement model and the calibration model are established to be used as an evaluation model to evaluate the measurement accuracy for the measuring system.

(2) The influence of hardware parameters on the measurement performance indexes of the system is analyzed and the corresponding constraint models for measurement performance are 
established.

(3) The corresponding influence laws between the hardware parameters and measurement performance are analyzed based on the constraint models using exhaustive search method and variable parameter method respectively to provide a reference for hardware parameters selection.

Henceforth, this paper is organized as follows: The flat contact R-test theoretical measurement model and calibration mode are established in Section 2. In Section 3, the hardware parameters and the performance indexes of the measurement system are defined, and the corresponding constraint models for measurement performance evaluation indexes are established. Subsequently, Section 4 introduces the optimal configuration methods of the geometric and precision parameters of hardware in detail. The case study for hardware configuration methods under certain requirements is verified and the performance evaluation results are analyzed in Section 5. Finally, some conclusions are drawn.

\section{The theoretical model of flat contact R-test}

\subsection{Structure description and parameter definition}

As shown in Fig. 1, the structure of the flat contact R-test mainly includes a precise master ball and 3 same evenly distributed flat contact displacement sensors. The master ball is mounted on the spindle of the machine tool by the tool holder, and it is required that the center of the master ball must be on the axis of the spindle. The sensors are perpendicular to each other in space and fixedly connected to the base("sensor nest"[25]) which is installed on the worktable. The top of the sensor is three contact planes perpendicular to the axis of the sensor.

Define the radius of the master ball as $R$, and the center points of the bottom as $S_{1_{-} 1}, S_{2_{-} 1}$ and $S_{3_{-} 1}$ respectively which is formed the reference plane of the R-test, the elevation angle between the sensor axis and the reference plane as $\alpha$, the angle between the projection lines of the sensor axis in the reference plane as $\beta$ (The default is $120^{\circ}$ ), and the radius of the circle $S_{1_{-}} S_{2_{-}} S_{3_{-} 1}$ as $\lambda$.

To simplify the calculation, the measurement coordinate system $\left(\mathrm{CSYS}_{\mathrm{M}}\right)$ is established with the intersection point of the three sensor axes as the origin $\mathrm{O}_{\mathrm{M}}$. The coordinate axes are parallel to the $\mathrm{X}, \mathrm{Y}$, and $\mathrm{Z}$ feed axes of the machine tool. The XOY coordinate plane is parallel to the reference plane $S_{1_{-} \mid} S_{2_{-}} S_{3_{-} 1}$, and the axis of the first sensor is lied in the XOZ plane.

Define the tangent point between the master ball and the top plane of the sensor as $P_{i}\left(x_{i}, y_{i}, z_{i}\right)$, $i=1,2,3$, the center points of the contact planes as $S_{i_{-} 0}$, and the reading of the contact displacement sensor as $l_{i}$ (The distance of $S_{i_{-}} S_{i_{-}}$). In the $\mathrm{CSYS}_{\mathrm{M}}$, the coordinates of the sphere center, the center point of the contact plane at the top of the sensor, the sensor position and the subpoint of the point $O_{\mathrm{M}}$ on the reference plane are defined as $P(x, y, z), S_{i_{-} 0}\left(x_{i_{-} 0} 0, y_{i_{-}} 0, z_{i_{-}}\right), S_{i_{-}}\left(x_{i_{-} 1}, y_{i_{-}} 1, z_{i_{-}}\right)$ and $O_{\mathrm{M}}^{\prime}\left(0,0, z_{i_{-}}\right)$respectively.

During the measuring process, the sphere center coordinates under the $\mathrm{CSYS}_{\mathrm{M}}$ at any position can be calculated according to the readings $l_{i}$ of the three sensors. 


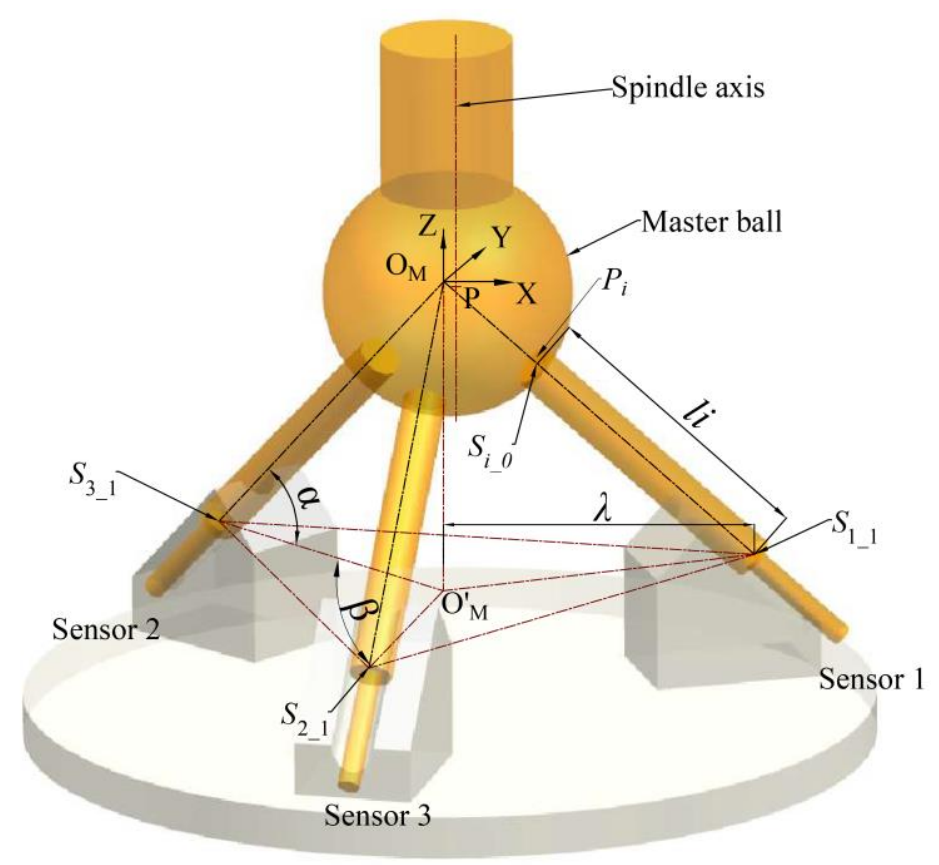

Fig. 1 The schematic diagram of flat contact R-test model.

\subsection{The theoretical measurement model}

To achieve the goal of using the measuring instrument to characterize the coordinates of points in space, a measurement model between the sensor readings and the coordinates of the sphere center must be established. According to the relationship of the point $S_{i_{-} 0}$ on the line $O_{\mathrm{M}} S_{i_{-} 1}$, the following position expression can be constructed as

$$
\overrightarrow{S_{i_{-} 1} S_{i_{-} 0}}=l_{i} \cdot \frac{\overrightarrow{S_{i_{-} 1} O_{\mathrm{M}}}}{\left|\overrightarrow{S_{i_{-} 1} O_{\mathrm{M}}}\right|}, \quad i=1,2,3
$$

When the measuring instrument is in the ideal initial position, the sphere center is at the origin of the CSYS $\mathrm{M}_{\mathrm{M}}$, and the readings $l_{i}$ of the three sensors are all half-range $l_{0} / 2$. The center of the detection plane $S_{i_{-} 0}$ is the tangent point between the ball and the detection plane, and there is a geometric relationship as

$$
\left|\overrightarrow{O_{M}^{\prime} P}-\left(\overrightarrow{O_{M}^{\prime} S_{i_{-} 1}}+\overrightarrow{S_{i_{-} 1} S_{i_{-} 0}}\right)\right|=\left|\overrightarrow{S_{i_{-} 0} P}\right|=R, i=1,2,3
$$

Assuming that the normal vector of the detection plane of the three sensors is $\boldsymbol{n}_{i}=\left(a_{i}, b_{i}, c_{i}\right)$, $i=1,2,3$, the normal equation of the three detection planes can be expressed as

$$
a_{i} X+b_{i} Y+c_{i} Z-\left(a_{i} x_{i_{-} 0}+b_{i} y_{i_{-} 0}+c_{i} z_{i_{-} 0}\right)=0, i=1,2,3
$$

Since the distance between the sphere center and the detection plane is always $R$, assuming that the detection plane and the axis of the sensor is keeping in an ideal vertical state, the expression of the coordinates of the sphere center $P$ concerning the sensor's reading $l_{i}$ is obtained as 


$$
\frac{\left|\boldsymbol{n}_{i} \cdot \overrightarrow{S_{i_{-} 0} P}\right|}{\left|\boldsymbol{n}_{i}\right|}=R, i=1,2,3
$$

where

$$
\boldsymbol{S}_{i_{-} 0}=\frac{\sqrt{x_{i_{-} 1}^{2}+y_{i_{-} 1}^{2}+z_{i_{-} 1}^{2}}-l_{i}}{\sqrt{x_{i_{-} 1}^{2}+y_{i_{-} 1}^{2}+z_{i_{-} 1}^{2}}} \boldsymbol{S}_{i_{-} 1}
$$

Then the theoretical measurement model can be expressed as Eqs.(6), which is used as the follow-up measurement accuracy evaluation model for measuring instrument.

$$
\left\{\begin{array}{l}
\frac{\left|a_{i}\left(x-x_{i_{-} 1}+x_{i_{-}} \cdot K_{i}\right)+b_{i}\left(y-y_{i_{-} 1}+y_{i_{-}} \cdot K_{i}\right)+c_{i}\left(z-z_{i_{-} 1}+z_{i_{-}} \cdot K_{i}\right)\right|}{\sqrt{a_{i}^{2}+b_{i}^{2}+c_{i}^{2}}}-R^{2}=0 \\
K_{i}=l_{i} / \sqrt{x_{i_{-}}^{2}+y_{i_{-}}^{2}+z_{i_{-} 1}^{2}}
\end{array}, i=1,2,3\right.
$$

And the coordinates of the sphere center can be calculated according to $a_{i}, b_{i}, c_{i}, x_{i_{-} 1}, y_{i_{-} 1}, z_{i_{-} 1}$ and the sensor reading $l_{i}$ based on the measurement Eqs. (6), the solution model is

$$
\left\{\begin{array}{l}
f_{2}(x, y, z)= \\
\frac{\left|a_{i}\left(x-x_{i_{-} 1}+x_{i_{-} 1} \cdot K_{i}\right)+b_{i}\left(y-y_{i_{-} 1}+y_{i_{-}} \cdot K_{i}\right)+c_{i}\left(z-z_{i_{-} 1}+z_{i_{-}} \cdot K_{i}\right)\right|}{\sqrt{a_{i}^{2}+b_{i}^{2}+c_{i}^{2}}}-R^{2} \\
=0 \\
G(x, y, z)=\sum_{n=1}^{3} f_{2}^{2}(x, y, z)
\end{array}, i=1,2,3\right.
$$

\subsection{The calibration model}

The determination of the sensor position is a necessary condition for solving the spherical center coordinates. However, it can be known from the structural characteristics of the R-test that the position coordinates of the sensors are directly affected by the installation errors of the sensors, which include the installation angle deviation $\Delta \alpha_{i}$ between the sensor axis and the Z-axis of the CSYS $_{\mathrm{M}}$, and the angle deviation $\Delta \beta_{i}$ projected on the reference plane of the three sensor axes. In general, the sensor installation error can be eliminated during the instrument calibration process, and the most common calibration methods mainly include two methods: preliminary calibration and on-machine calibration ${ }^{[16]}$. Moving the feed axis of the calibration equipment accurately in the $\mathrm{CSYS}_{\mathrm{M}}$, the sphere center is located at 6 arbitrary calibration points $P_{j}\left(x_{j}, y_{j}, z_{j}\right), j=1,2, \ldots, 6$ within the measuring range of the sensor. Substituting the calibration point coordinates into the Eqs. (6), the coordinates of the normal vector $\boldsymbol{n}_{i}$ and the position $S_{i_{-} 1}$ of the sensor detection planes are solved. The system of equations can be solved using Self-adaptive Different Evolution(SaDE) to avoid the problem of multiple solutions. The calibration model when solving the normal vector and position of the sensor detection planes can be set as 


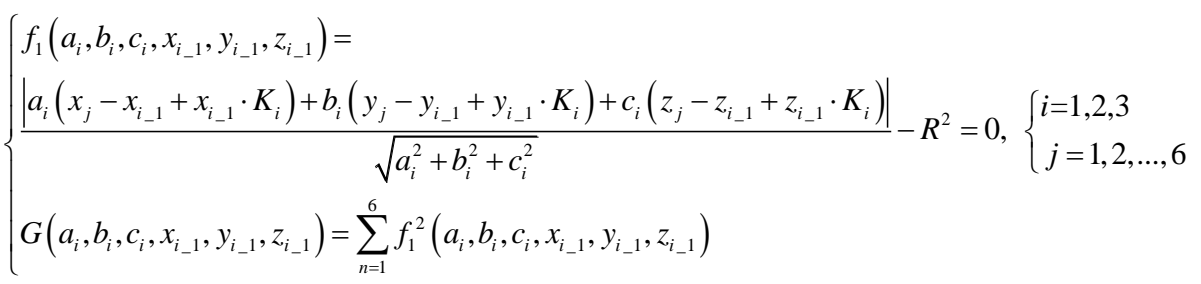

\section{Definition of hardware parameters and performance indexes of measurement system}

\subsection{The definition of hardware parameters}

It can be seen from Eqs.(7) and Eqs.(8) that the error terms affecting the performance of the measurement system can be summarized as shown in Tab. 1. The errors caused by hardware installation including $\Delta \alpha, \Delta \beta, \Delta S_{i_{-} 1}$ and $\Delta \boldsymbol{n}_{i}$ can be directly eliminated during the calibration process. And ensure the precision of the calibration points as much as possible to eliminate the impact of calibration errors on the final measurement accuracy through a high-precision calibration equipment.

When calibration is completed, the inherent errors of the hardware have the greatest impact on the measurement accuracy of the measurement system. The inherent errors of hardware are directly reflected in the geometric parameters which include the range of the sensor $l_{0}$ and the radius of the ball $R$ and precision parameters which include the precision of the sensor reading $\Delta L_{i}$, $i=1,2,3$ and the ball radius $\Delta R$. The geometric parameters affect the spatial movement range of the sphere center, and the precision parameters directly affect the solution accuracy of the sphere center coordinates, which in turn affects the accuracy of the R-test error measurement data. In addition, it can be known from the structural characteristics of the R-test that the radius of the sensor detection plane (Defined as $r_{i}, i=1,2,3$ ) are also one of the important factors affecting the moving range of the sphere center.

Tab. 1 Error term affecting the performance of flat contact R-test.

\begin{tabular}{cc}
\hline \multicolumn{1}{c}{ Definition } & Value \\
\hline The installation angle deviation between the sensor axis and the & $\Delta \alpha$ \\
The angle deviation projected on the reference plane of the three & $\Delta \beta$ \\
sensor axes. & $\Delta S_{i_{-} 1, i=1,2,3}$ \\
The installation position error of sensors. & $\Delta \boldsymbol{n}_{i}, i=1,2,3$ \\
The direction deviation of the sensor detection plane normal vector. & $\left(\Delta x_{j}, \Delta y_{j}, \Delta z_{j}\right), j=1,2, \ldots, 6$ \\
The reading error of the sensors. & $\Delta L_{i}, i=1,2,3$ \\
The radius error of the ball. & $\Delta R$
\end{tabular}




\subsection{The definition of measurement system performance indicators}

The performance evaluation indexes of the R-test mainly include measuring stability, measuring space and measurement accuracy. To improve the adaptability and safety of the flat contact R-test, it is necessary to maximize the measuring stability and the measuring space at the same time under the premise of ensuring measurement accuracy.

(1) Measuring stability

The measuring stability characterizes the magnitude of the sensor's reading change $\Delta \boldsymbol{l}_{i}, i=1,2,3$ when there is a small amount of position change at the sphere center. The relational expression is

$$
\boldsymbol{\Delta} \boldsymbol{P}=\boldsymbol{J} \cdot \Delta \boldsymbol{l}_{i}, \quad i=1,2,3
$$

where $\boldsymbol{\Delta} \boldsymbol{P}=(\Delta x, \Delta y, \Delta z)$, and the Jacobian matrix $\boldsymbol{J}$ represents the mapping relationship between $\Delta \boldsymbol{P}$ and $\Delta \boldsymbol{l}_{i}$, which is only related to the installation angle of the sensor ${ }^{[16]}$. The smaller amplitude of $\Delta l_{i}$, the better measuring stability.

(2) Measuring space

As shown in Fig. 2, the measuring space represents a range of movement of the sphere center within the range of the sensors, is represented by $S$. According to the structural characteristics of the flat contact R-test, the movement range of the sphere center should be limited to a cylinder with a ground radius of $r_{i}$ and a height of $l_{0}$ for a single sensor during the measurement process, and then the measuring space of the R-test is the intersection of three angled cylinders, which should be larger than the required measurement range. In theory, the larger the measuring space, the larger the machine tool error range that the instrument can measure and the higher the safety. However, after the structural parameters of the measuring device are determined, the measuring space is limited by the sensor range, the sensor's detection plane radius and the ball radius. So the range of the sensor and the radius of the ball needs to be optimized for a specific measuring space requirement, to avoid waste of space.

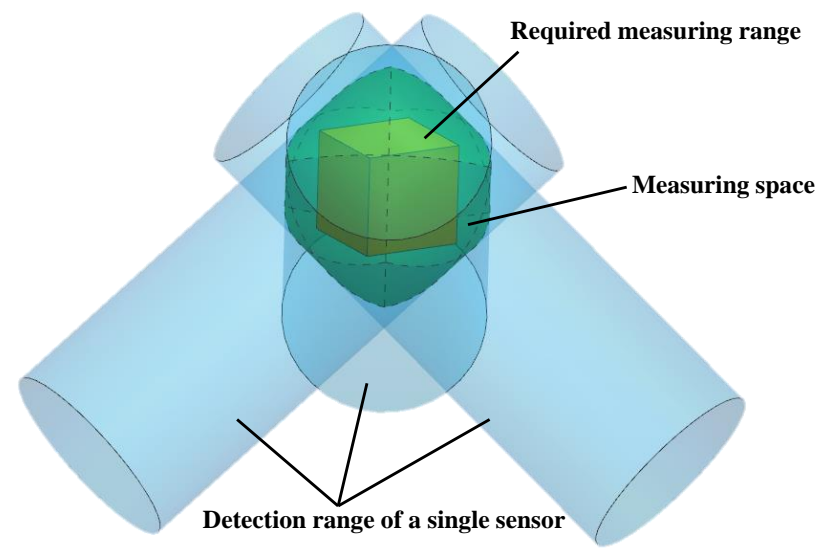

Fig. 2 Schematic diagram of measurement space.

(3) Measurement accuracy

The measurement accuracy is defined as the solution accuracy of the sphere center coordinates calculated by the sensor readings during the calibration process, which is mainly affected by 
positioning accuracy of the calibration equipment, the precision of the sensor reading $\Delta L_{i}$ and the ball radius $\Delta R$, can be expressed by the calculation uncertainty of the spherical center coordinates $u(x), u(y), u(z)$. Measurement accuracy directly affects the accuracy and validity of subsequent error measurement data, so it needs to be prioritized.

\subsection{The constraint model of measurement performance indexes for the R-test}

(1) The constraint model of measuring stability

To quantify the relationship between measuring stability and instrument hardware parameters, the constraint models are established based on the movement law of the center of the sphere at the origin of the MCS under ideal conditions. As shown in Fig. 3, the origin of the MCS is at the half-range of the three sensors in an ideal initial situation. The tangent points between the ball and the detection planes of the sensors are just the center point $S_{i_{-} 0}$ of the detection planes, and the center distance of the sensor $\lambda=\left(\mathrm{R}+l_{0} / 2\right) \cdot \cos \alpha$. At this time, the constraint model for the measuring stability can be obtained as Eqs.(10) according to Eq. (2). Based on the Eqs.(10), the installation angle requirement for maximum stability can be obtained by solving the second-order condition number of the matrix formed by the partial derivative of the sphere center coordinates relative to the sensor reading ${ }^{[16]}$, and the detailed solution process will not be repeated here.

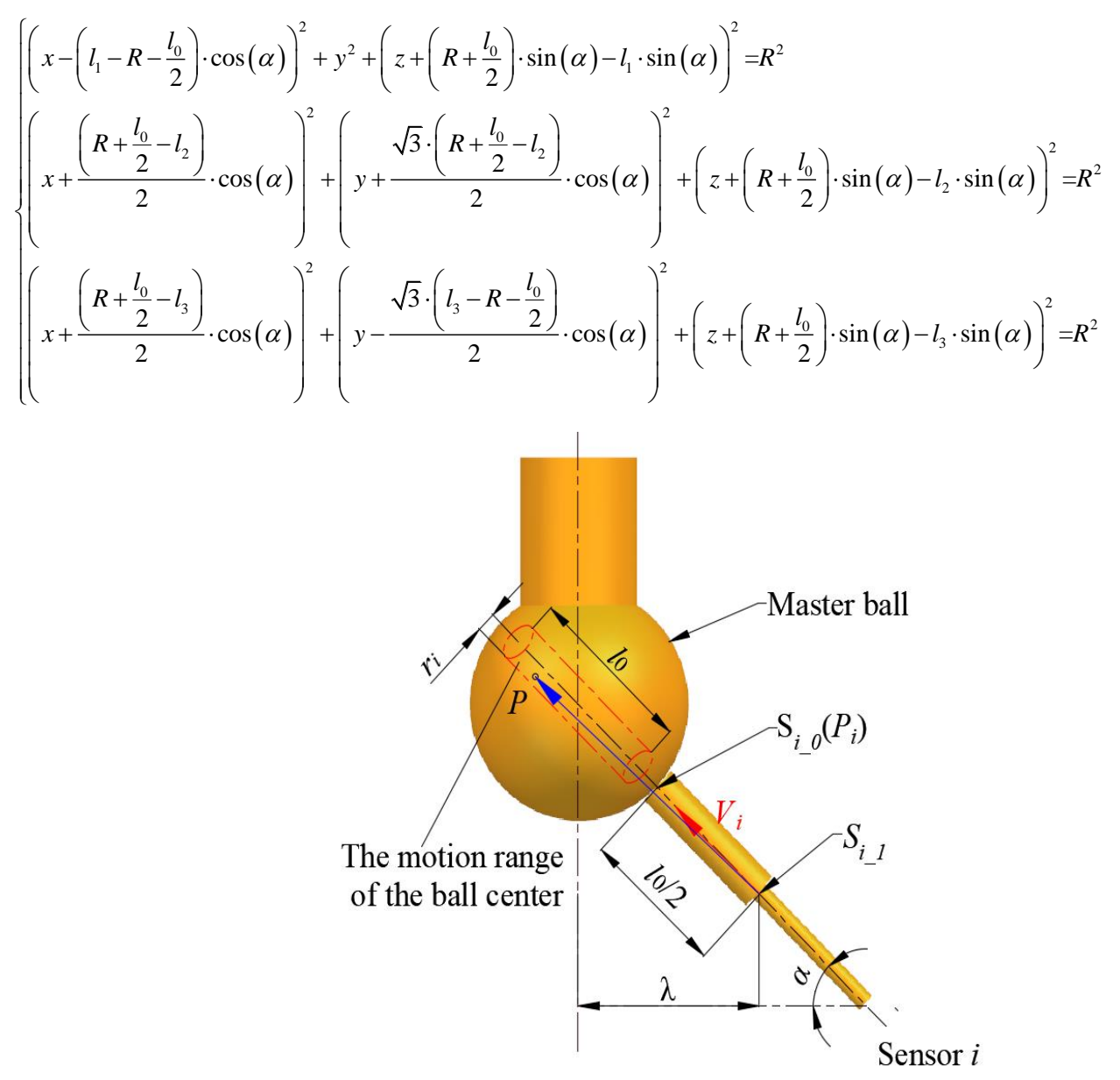

Fig. 3 Schematic diagram of the range of movement of the center of the sphere at the measurement origin.

(2) The constraint model of measuring space

As for the measuring space, when the sensor installation angles are certain, it is simultaneously 
affected by the sensor range, the sensor detection plane radius and the ball radius. When the positions of the sensors are fixed, define the normal vector of the detection plane at the bottom plane of the sensor as $\boldsymbol{V}_{i}, i=1,2,3$, and the distance from the center of the sphere to the bottom plane of the sensor as $d_{i}, i=1,2,3$. According to the spatial geometric position relationship between the sensors and the ball, the constraint equation can be obtained

$$
\left\{\begin{array}{l}
\left|\overrightarrow{S_{i_{-}} P} \times V_{i}\right| \leq\left|V_{i}\right| \cdot r_{i} \\
R \leq d_{i} \leq R+l_{0}, i=1,2,3 \\
0<r_{i} \leq R
\end{array}\right.
$$

Therefore, the influence relationship between measuring space and hardware parameters can be expressed as Eqs.(12) to Eqs.(14)

$$
\begin{aligned}
& \left\{\begin{array}{l}
\left|\begin{array}{cc}
y & z+\lambda \cdot \tan \alpha \\
0 & \lambda \cdot \tan \alpha
\end{array}\right|^{2}+\left|\begin{array}{cc}
x-\lambda & z+\lambda \cdot \tan \alpha \\
-\lambda & \lambda \cdot \tan \alpha
\end{array}\right|^{2}+\left|\begin{array}{cc}
x-\lambda & y \\
-\lambda & 0
\end{array}\right|^{2}-\lambda^{2} \cdot r^{2} \cdot \sec ^{2} \alpha \leq 0 \\
R \leq \frac{\mid x-z \cdot \tan \alpha-\lambda \cdot \sec ^{2} \alpha}{\sec \alpha} \leq R+l_{0} \\
0<r_{1} \leq R
\end{array}\right.
\end{aligned}
$$



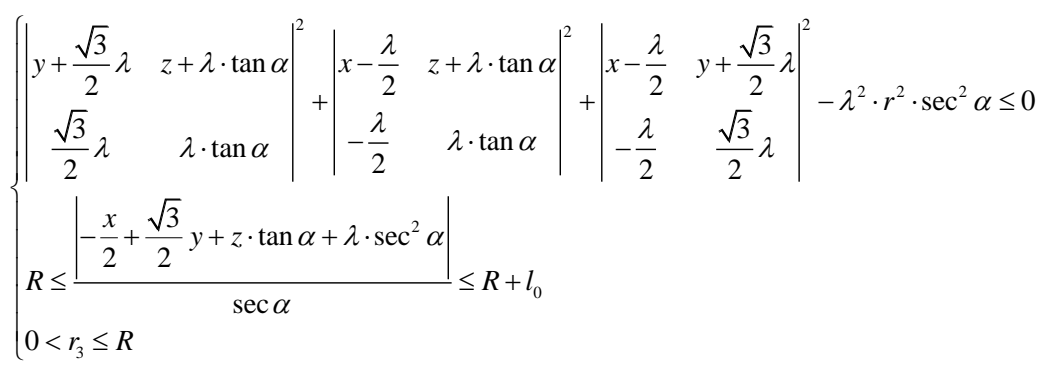

(3) The constraint model of measurement accuracy

Since the measurement accuracy is mainly affected by the precision of the equipment hardware itself in the calibration process and the sphere center solution process. In order to study the propagation of various actual errors in the process of solving the spherical center coordinates, and analyze their influence on the final measurement accuracy, the degree of influence of various influencing factors on the measurement performance of the measuring instrument can be calculated through the Guide to the Expression Uncertainty in Measurement(GUM) and Monte Carlo Method(MCM) based on the Eqs. (6). The analysis of the degree of influence of specific hardware precision parameters on measurement accuracy will be given in section 4.2 and the analysis results will be given in section 5.1 for a specific case. 


\section{Optimized configuration of the hardware parameters based on the constraint models}

It can be seen from the above analysis that the geometric parameters and precision parameters of the hardware directly affect the measurement performance of the R-test. Therefore, the optimized configuration methods for the hardware parameters based on the constraint models are proposed, which guide the customized configuration of the R-test in practical applications and avoid waste of hardware range and precision. The optimization configuration process is shown in Fig. 4.

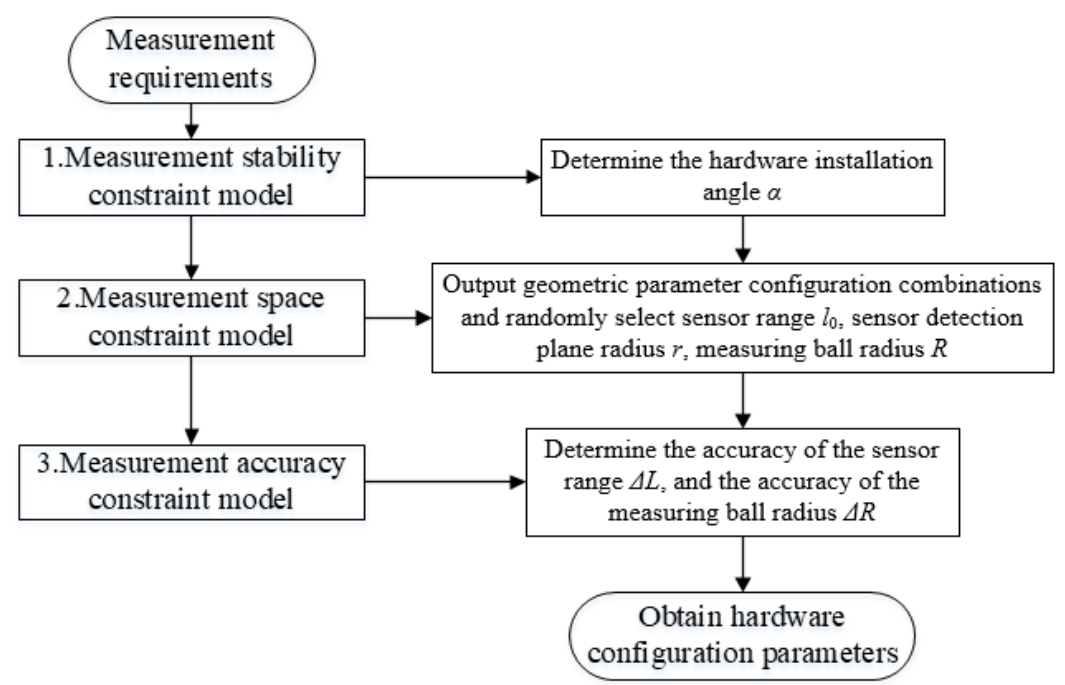

Fig. 4 Flow chart of hardware parameter optimization based on constraint model.

\subsection{Determine sensor installation angle based on measuring stability constraint model}

It can be known from the optimization method in the literature [16] and Eqs.(10) that the measuring stability of the R-test is only related to the sensor elevation angle $\alpha$, and is not affected by the sensor range $l_{0}$ and the ball radius $R$. Therefore, the maximum measuring stability can be ensured by determining the parameter of the sensor elevation angle $\alpha$, and the maximum measuring stability can be obtained when $\alpha$ is $35.3^{\circ}$.

\subsection{The configuration optimization of hardware geometric parameters based on the measuring space constraint model}

After the value of $\alpha$ is fixed, the size of the measuring space is mainly limited by the hardware parameters which include sensor range $l_{0}$, the sensor detection plane radius $r_{i}$ and the ball radius $R$ according to Eqs.(12) to Eqs.(14). To study the selection of hardware configuration for certain measuring space requirements, an optimal configuration method based on the exhaustive search is proposed. Assuming that the three sensors are of the same type, the detection plane $\operatorname{radius}\left(r_{1}=r_{2}=r_{3}=r\right)$ and range are the same. According to the R-test measuring characteristics and the five-axis machine tool RTCP movement principle, the sensor range needs to ensure that the moving range of the ball is greater than the maximum error of the rotating shaft (Generally no 
more than $0.1 \mathrm{~mm}$ ), and a safety margin needs to be set to move the sphere center to the origin of the $\mathrm{CSYS}_{\mathrm{M}}$ more conveniently. The selection of the radius of the ball needs to consider the range of the sensor and the radius of the detection plane to a certain extent.

Defined the certain measuring space as $\left[x_{\min }, x_{\max }\right]^{*}\left[y_{\min }, y_{\max }\right]^{*}\left[z_{\min }, z_{\max }\right]$, the sensor range as $\left[l_{\min }, l_{\max }\right]$, the radius of the detection plane as $\left[r_{\min }, r_{\max }\right]$, and the ball radius as $\left[R_{\min }, R_{\max }\right]$. The preset range of hardware parameters can be roughly set based on historical experience, and the range is as large as possible. So the above optimization configuration method can be expressed Step are shown as Fig. 5:

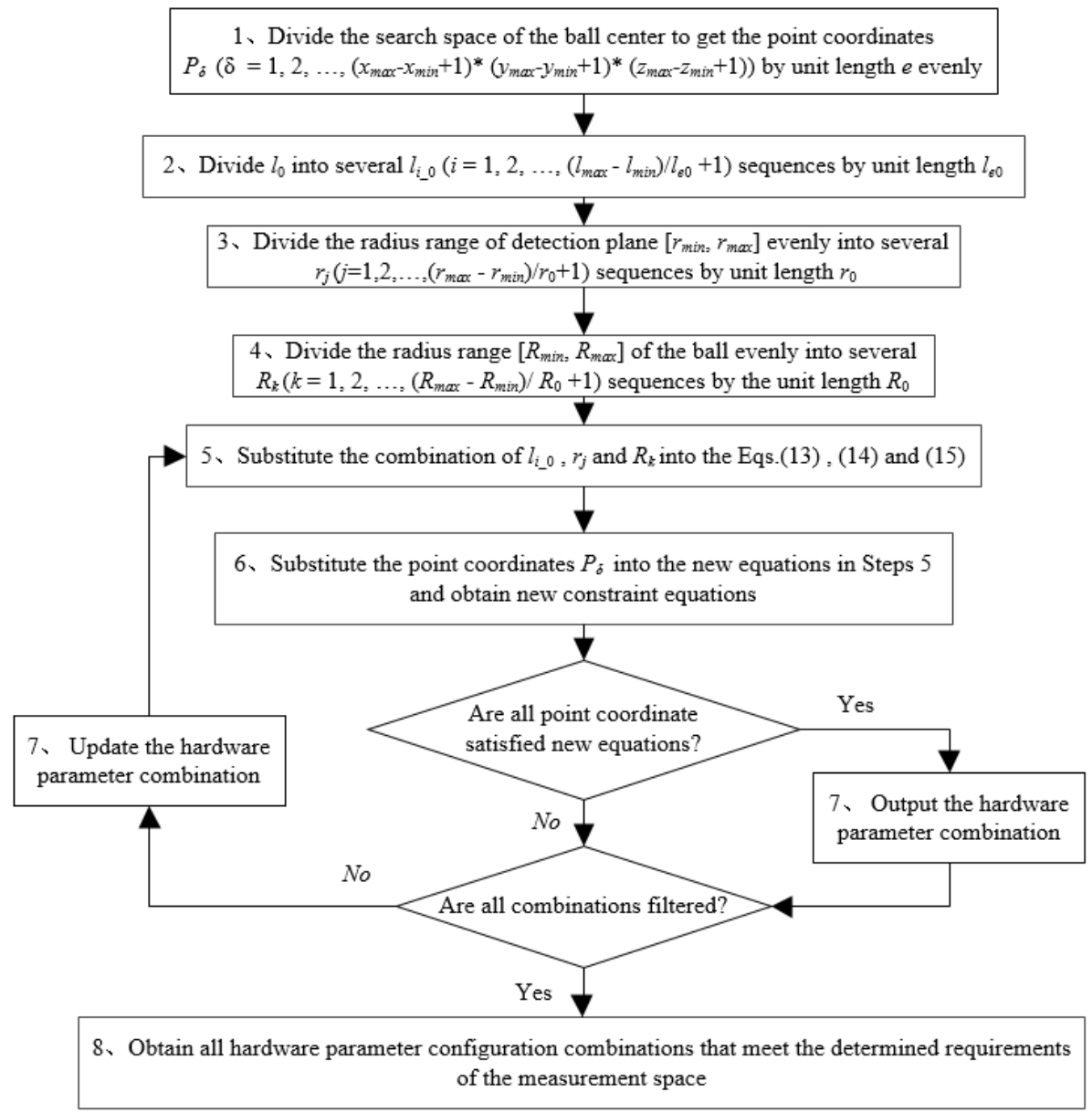

Fig. 5 Flow chart of the optimized configuration of sensor range and the sphere radius.

Step 1: Divide the search interval into several spherical center point coordinates $P_{\delta}(\delta=1,2, \ldots$, $\left(x_{\max }-x_{\min }+1\right) *\left(y_{\max }-y_{\min }+1\right) *\left(z_{\max }-z_{\min }+1\right)$ by unit length $e$ evenly;

Step 2: Divide the sensor range $\left[l_{\min }, l_{\max }\right]$ evenly into several $l_{i_{-} 0}\left(i=1,2, \ldots,\left(l_{\max }-l_{\min }\right) / l_{e 0}+1\right)$ sequences by unit length $l_{e 0}$;

Step 3: Divide the radius range of detection plane $\left[r_{\min }, r_{\max }\right]$ evenly into several $r_{j}$ $\left(j=1,2, \ldots,\left(r_{\max }-r_{\min }\right) / r_{0}+1\right)$ sequences by unit length $r_{0}$;

Step 4: Divide the radius range $\left[R_{\min }, R_{\max }\right]$ of the ball evenly into several $R_{k}\left(k=1,2, \ldots,\left(R_{\max }\right.\right.$ - $\left.R_{\min }\right) / R_{0}+1$ ) sequences by the unit length $R_{0}$;

Step 5: Substitute the hardware parameter combination of $l_{i_{-} 0}, r_{j}$ and $R_{k}$ into the Eqs.(12), 
Eqs.(13) and Eqs.(14) at the same time and obtain new constraint equations corresponding to the combination;

Step 6: Substitute the point coordinates $P_{\delta}$ into the new equations in Step 5 and judge whether all point coordinates meet constraint equations.

Step 7: If all the point coordinates in Step 6 meet the constraint equations, output the hardware parameter combination; if not, update the combination value, and repeat Step 5 and Step 6 until all combinations are filtered.

Step 8: Obtain all hardware parameter configuration combinations that meet the certain requirements of the measuring space.

\subsection{Optimal configuration of hardware precision parameters based on the measurement accuracy constraint model}

For measuring instrument with certain hardware geometric parameters, it can be seen that the precision parameters of hardware which include the reading precision $\Delta L_{i}$ of the sensor and the radius precision $\Delta R$ of the ball, mainly affect the solution accuracy of the sphere center coordinates and directly affect the final measuring accuracy of the measuring instrument according to the measuring Eqs. (6) in Section 2.2. In general, the higher the precision of the hardware, the higher the cost, and when a certain precision limitation is reached, the price of hardware will increase geometrically. However, the precision selection of hardware needs to be certain by actual measuring requirements in actual engineering applications. So to ensure the accuracy of the measuring instrument and avoid the waste of hardware precision, the precision of the sensors and the ball must be reasonably configured.

Assuming that the three sensors have the same indication precision $\left(\Delta L_{1}=\Delta L_{2}=\Delta L_{3}=\Delta L\right)$, the method of variable parameters is used to analyze and observe the influence of the precision of the sensor's indication and the ball radius on the final measuring accuracy of the measuring instrument based on the Eqs. (6). And the sensitivity coefficient is used to characterize the degree of influence more intuitively. To calculate the sensitivity coefficient based on the R-test complex model more accurately and quickly, the understanding of the evaluation model is reduced to an empirical first-order Taylor series expansion based on the measured sensitivity coefficient ${ }^{[26]}$, and the sensitivity coefficient is also defined based on the variable parameter method, the calculation Step are as follows:

Step 1: Substitute the calibration point coordinates $P_{j}$ and corresponding sensor readings $l_{i}$ into Eq.(8) and calculate the normal vector $\boldsymbol{n}_{i}$ of the detection plane of the three sensors and the sensor position coordinates $S_{i_{-}}$;

Step 2: Substitute $\boldsymbol{n}_{i}$ and $S_{i_{-} 1}$ obtained in Step 1 into Eqs. (7) to obtain the coordinates of the sphere center under ideal conditions at any position;

Step 3: Substitute the indication error of sensors $\Delta L$ and the radius error of ball $\Delta R$ into the Eq.(7), the sensitivity solution model of the sphere center coordinates change $\boldsymbol{\Delta} \boldsymbol{P}$ relative to the sensor precision $\Delta L$ and the sphere radius precision $\Delta R$ is obtained: 


$$
\Delta \boldsymbol{P}=f_{4}\left(\boldsymbol{n}_{i}, S_{i_{-}}, \Delta L, \Delta R\right), i=1,2,3
$$

Step 4: Define the reasonable change interval of $\Delta L$ as $\pm \delta$, the change step length is $e$, and the number of step lengths is $n$;

Step 5: Calculate the indication value $\Delta L_{m}(m=1,2, \ldots, n)$ corresponding to each step respectively;

Step 6: Ensure that the rest of the input is unchanged, and calculate the change in sphere center coordinates ( $\Delta x_{L m}, \Delta y_{L m}, \Delta z_{L m}$ ) corresponding to $\Delta L_{m}$ according to the Eq.(15);

Step 7: Calculate the change in sphere center coordinates $\left(\Delta x_{R m}, \Delta y_{R m}, \Delta z_{R m}\right)$ corresponding to $\Delta R_{m}$ according to the calculation principle from step 4 to step 6;

Step 8: Obtain the regular distribution diagram between hardware precision parameters $(\Delta L$ and $\Delta R)$ and the solution accuracy of the sphere center coordinates;

Step 9: Calculate the mean value of the change in the center of the sphere by Eq.(16)

$$
\begin{aligned}
& \left(\overline{\Delta x_{L m}} \overline{\Delta y_{L m}} \quad \overline{\Delta z_{L m}}\right)= \\
& \left(\frac{\sum_{m=1}^{n}\left|\frac{\Delta x_{L m}}{-\delta+e \cdot m}\right|}{n}\left|\frac{\sum_{m=1}^{n}\left|\frac{\Delta y_{L m}}{-\delta+e \cdot m}\right|}{n}\right| \frac{\sum_{m=1}^{n}\left|\frac{\Delta z_{L m}}{-\delta+e \cdot m}\right|}{n}\right)
\end{aligned}
$$

Step 10: Calculate the mean value of the change in the sphere center coordinates relative to $\Delta R$ using the method in Step (4) to Step (7) as shown in Eq. (17)

$$
\begin{aligned}
& \left(\overline{\Delta x_{R m}} \frac{\overline{\Delta y_{R m}}}{\overline{\Delta z_{R m}}}\right)= \\
& \left(\frac{\sum_{m=1}^{n}\left|\frac{\Delta x_{R m}}{-\delta+e \cdot m}\right|}{\frac{\sum_{m=1}^{n} \mid \frac{\Delta y_{R m}}{-\delta+e \cdot m}}{n}}\left|\sum_{m=1}^{n}\right| \frac{\Delta z_{R m}}{-\delta+e \cdot m} \mid\right)
\end{aligned}
$$

Step 11: Normalization and dimensionless processing, to obtain the sensitivity coefficients $c_{L}$ and $c_{R}$ corresponding to $\Delta L$ and $\Delta R$ respectively, and then provide reference and guidance for the precision selection of hardware according to the precision influence law and the corresponding sensitivity coefficients of the two.

\section{Case verification and result analysis}

\subsection{Case calculation}

Take specific measurement requirements (The measuring space is $0.3 * 0.3 * 0.3 \mathrm{~mm}^{3}$, and the measurement accuracy is $4 \mu \mathrm{m}$ ) as an example. The configuration method in section 4.2 is used to realize the configuration of hardware geometric parameters, and the method in section 4.3 is used to realize the configuration of hardware precision parameters.

(1) Hardware geometric parameter configuration

In general, the geometric error of the rotary axis of a five-axis machine tool does not exceed $0.3 \mathrm{~mm}$. Therefore, the moving range of the sphere center $x, y, z$ are all set as $[-0.3 \mathrm{~mm}, 0.3 \mathrm{~mm}]$. The initial preset calculation parameters are set as shown in Tab. 2. According to Step1 to Step 8 Section 4.2, 607620 group hardware configuration parameters can be configured for the measuring space range $[-0.3 \mathrm{~mm}, 0.3 \mathrm{~mm}]$ are shown in Table 2. 
Tab. 2 The table of the initial preset calculation parameters.

\begin{tabular}{cc}
\hline Preset parameters & Values $(\mathrm{mm})$ \\
\hline The given measuring space range & {$[-0.3,0.3]$} \\
$e$ & 0.1 \\
$l_{e 0}$ & 0.1 \\
$r_{0}$ & 0.5 \\
$R_{0}$ & 1 \\
{$\left[l_{\min }, l_{\max }\right]$} & {$[0.5,20]$} \\
{$\left[r_{\min }, r_{\max }\right]$} & {$[2.5,10]$} \\
{$\left[R_{\min }, R_{\max }\right]$} & {$[10,50]$} \\
\hline
\end{tabular}

Tab. 3 The table of hardware parameter configuration combinations for the measuring space $[-0.3 \mathrm{~mm}, 0.3 \mathrm{~mm}]$.

\begin{tabular}{cccc}
\hline \multirow{2}{*}{ Number } & \multicolumn{2}{c}{ Configuration combination of hardware parameter combinations $(\mathrm{mm})$} \\
\cline { 2 - 4 } & $l_{0}$ & $r$ & $R$ \\
\hline 1 & 0.6 & 2.5 & 10 \\
2 & 0.6 & 2.5 & 11 \\
$\ldots$ & 0.6 & 2.5 & 12 \\
$\ldots$ & $\ldots$ & $\ldots$ & $\ldots$ \\
$i-1$ & 1.1 & 3.5 & 14 \\
$i$ & 1.1 & 3.5 & 15 \\
$\ldots$ & 1.1 & 3.5 & 16 \\
607619 & $\ldots$ & $\ldots$ & $\ldots$ \\
607620 & 20 & 10 & 49 \\
\hline
\end{tabular}

(2) Hardware precision parameter configuration

Considering that the precision of the three sensors is the same, the contour diagram between the accuracy of the X-direction, $\mathrm{Y}$-direction, and Z-direction coordinate of the sphere center and the precision of the sensor and the ball can be calculated according to Eq.(15), shown in Fig. 6, Fig. 7, and Fig. 8 respectively. And the sensitivity coefficient of the influence of the precision of sensor indication and the ball radius on the solution accuracy of the sphere center coordinates is shown in Fig. 9 according to Step 1 to Step 10 in Section 4.3. The precision configuration of the sensor and the ball under any required measuring accuracy can be chosen according to the configuration influence law, and the precision of the sensor's indication needs to be controlled mainly. 


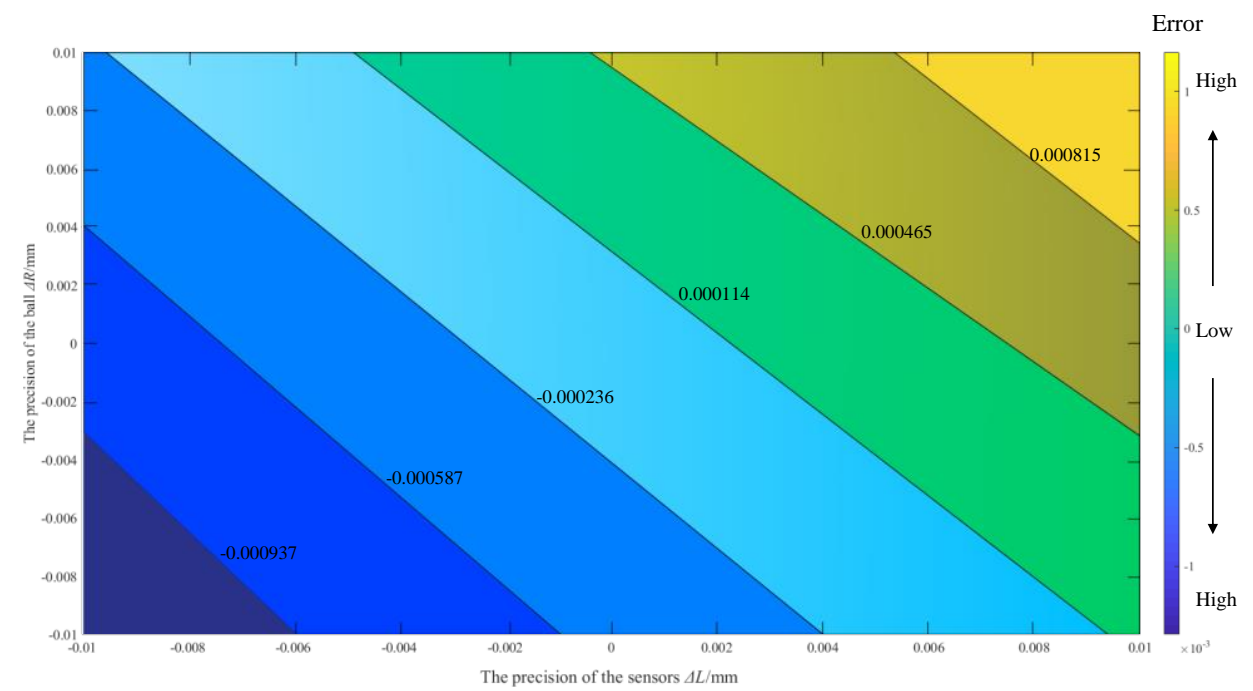

Fig. 6 The diagram of the relationship between the calculation accuracy of the sphere center X-direction coordinate and the precision parameters of the hardware.

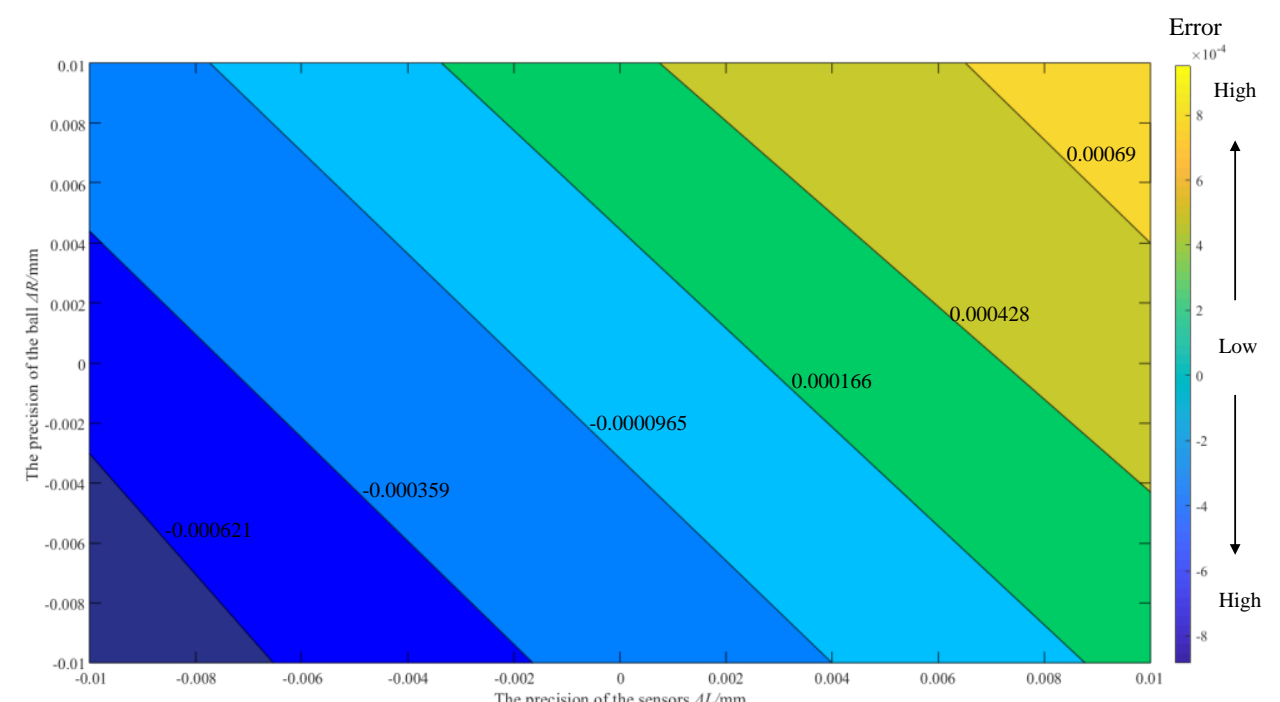

Fig. 7 The diagram of the relationship between the calculation accuracy of the sphere center Y-direction coordinate and the precision parameters of the hardware.

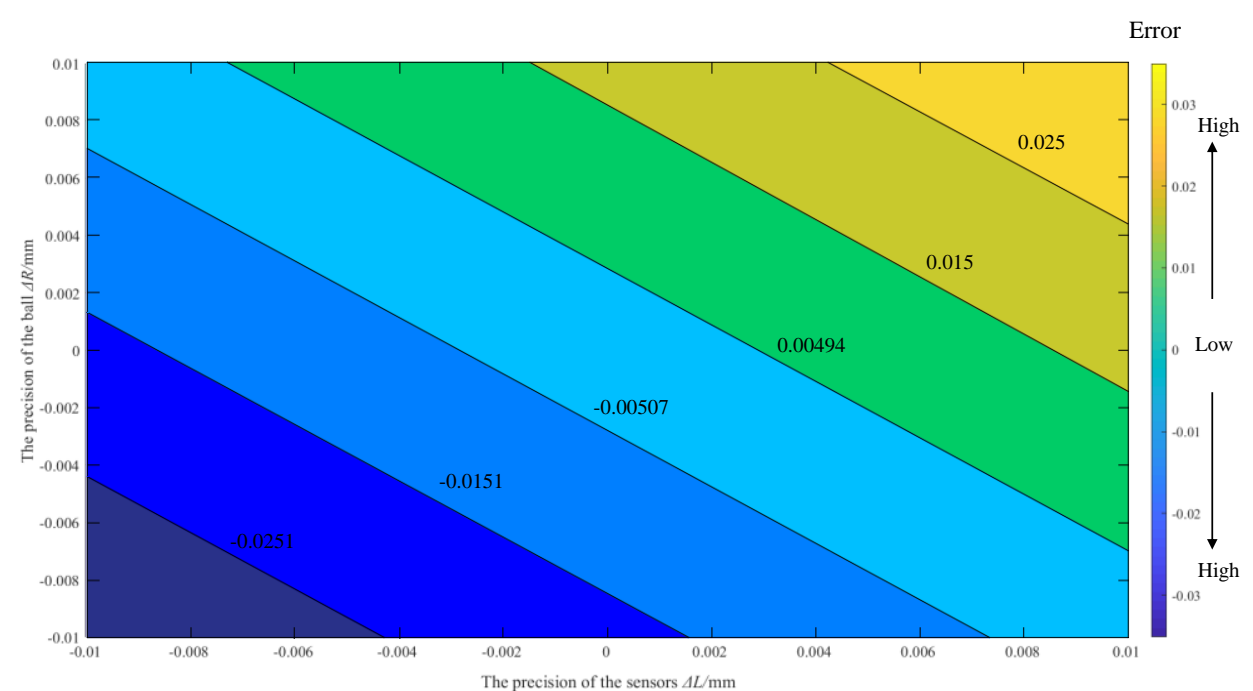


Fig. 8 The diagram of the relationship between the calculation accuracy of the sphere center Z-direction coordinate and the precision parameters of the hardware.

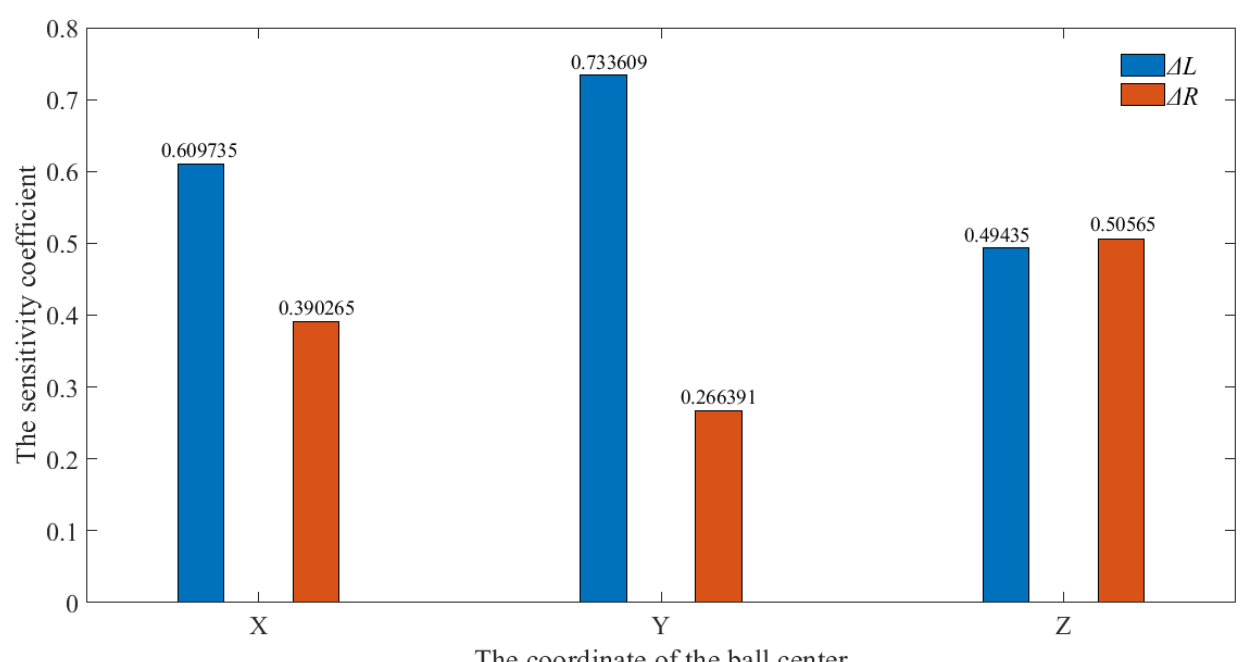

Fig. 9 The sensitivity coefficient of the influence of hardware precision on the solution accuracy of sphere center coordinates.

\subsection{Hardware selection and prototype development}

Configure the hardware parameters of the measurement requirements according to the measurement requirements and the analysis results in Section 5.1:

(1) The sensor range is selected as $1 \mathrm{~mm}$ (The maximum stroke $1.1 \mathrm{~mm}$, the and the maximum sampling frequency is approximately $400 \mathrm{~Hz}$ ), the detection plane radius of the sensor is $3.5 \mathrm{~mm}$, and select $15 \mathrm{~mm}$ for the radius of the ball;

(2) The precision of the sensor's indication is selected to be $1 \mu \mathrm{m}$, and the precision of the ball radius is controlled to be within $2 \mu \mathrm{m}$.

Select KEYENCE GT-S1 contact displacement sensor and D6L50M5 standard ceramic ball to develop a prototype (As shown in Fig. 10), and the R-test software is developed using Qt.

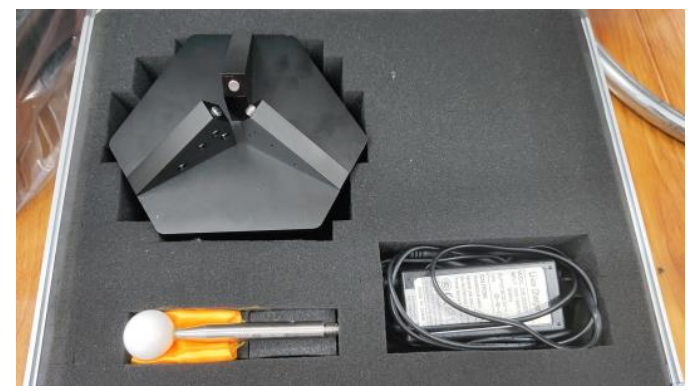

Fig. 10 The picture of the developed prototype.

\subsection{The evaluation of measuring space}

The calibration experiment on a CMM, as shown in Fig. 11, and the positioning error of the CMM is controlled to be less than $0.5 \mu \mathrm{m}$. The calibration point coordinates and the corresponding sensor readings are shown in Tab. 4, and the normal vector of the sensor detection planes $\boldsymbol{n}_{i}$ and position coordinates $S_{i_{-} 1}$ calculated from Tab. 4 and Eqs.(6) are shown in Tab. 5. According to the spherical center coordinates calculation Eqs. (7), the actual range of the spherical center 
movement within the certain range of the sensor can be calculated as shown in Fig. 12, which is an approximate cube with the volume of $0.49318 \mathrm{~mm}^{3}$. It can be seen that the R-test with the selected hardware geometric parameters configured according to the method in Section 4.1 meets the measuring space requirements of $0.3 * 0.3 * 0.3 \mathrm{~mm}^{3}$.

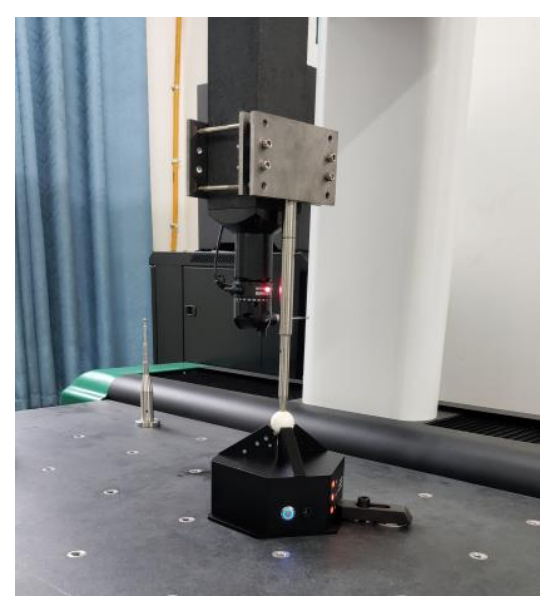

Fig. 11 The calibration scene of the R-test on the CMM.

Tab. 4 Calibration point coordinates and corresponding sensor readings.

\begin{tabular}{ccccccc}
\hline \multirow{2}{*}{$P_{j}$} & \multicolumn{3}{c}{ Calibration point coordinates } & \multicolumn{3}{c}{ Sensor readings } \\
\cline { 2 - 7 } & $x_{1 \_} 1$ & $y_{1 \_} 1$ & $z_{1} \_$ & $l_{1}$ & $l_{2}$ & $l_{3}$ \\
\hline 1 & 0.2 & 0.2 & 0.2 & 0.7151 & 0.8216 & 0.4467 \\
2 & -0.2 & -0.2 & -0.2 & 0.3991 & 0.2546 & 0.6688 \\
3 & -0.103 & -0.003 & -0.073 & 0.5619 & 0.4143 & 0.5569 \\
4 & -0.033 & -0.089 & 0.001 & 0.5120 & 0.5090 & 0.6357 \\
5 & 0.076 & -0.114 & 0.121 & 0.5115 & 0.6651 & 0.6794 \\
6 & -0.133 & -0.070 & -0.122 & 0.5015 & 0.3606 & 0.5902 \\
\hline
\end{tabular}

Tab. 5 The normal vector of the sensor detection planes $\boldsymbol{n}_{i}$ and position coordinates $S_{i_{-}}$.

\begin{tabular}{ccccccc}
\hline \multirow{2}{*}{ Sensor number $i$} & \multicolumn{3}{c}{$\boldsymbol{n}_{i}$} & \multicolumn{3}{c}{$S_{i_{-} 1}$} \\
\cline { 2 - 7 } & $a_{i}$ & $b_{i}$ & $c_{i}$ & $x_{i_{-}}$ & $y_{i_{-} 1}$ & $z_{i_{-} 1}$ \\
\hline 1 & -6.758 & 9.5608 & 8.2741 & 6.6537 & -7.3603 & -12.971 \\
2 & -14.759 & -0.5226 & -9.6063 & -11.686 & -0.20323 & -10.522 \\
3 & 10.14 & 14.766 & -13.99 & -0.19504 & 10.794 & -13.909 \\
\hline
\end{tabular}




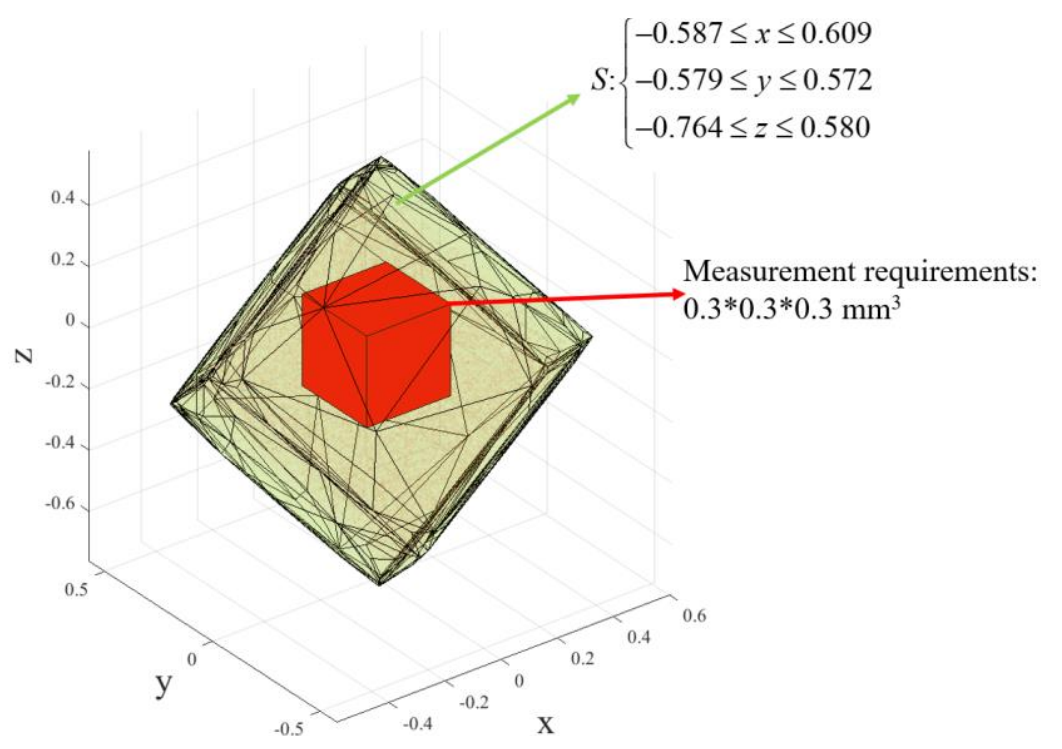

Fig. 12 Sphere center coordinate movement range within the sensor range.

\subsection{The evaluation of measurement accuracy}

To make the moving coordinates of the verification point more accurate, a laser interferometer is used to verify the calibration accuracy, which is shown in Fig. 13. Calculate the sphere center coordinates solution uncertainty based on the GUM and MCM, and compare with the actual verification point coordinate accuracy. The evaluation results of the sensor position calibration uncertainty are shown in Tab. 6 , and the calibration uncertainty of the sphere center is $2.141 \mu \mathrm{m}$, $2.763 \mu \mathrm{m}$ and $3.991 \mu \mathrm{m}$ respectively, which is consistent with the 1000 verification point errors in the actual calibration experiment as shown in Fig. 14. It can also be seen that the R-test with the selected hardware precision parameters configured according to the method in Section 4.2 meets the measurement requirements of $4 \mu \mathrm{m}$. Fig. 15 shows the on-machine measurement on the MIKRON machine tools using the prototype, and the measurement results show that the performance of the protype can meet the required measurement requirements.

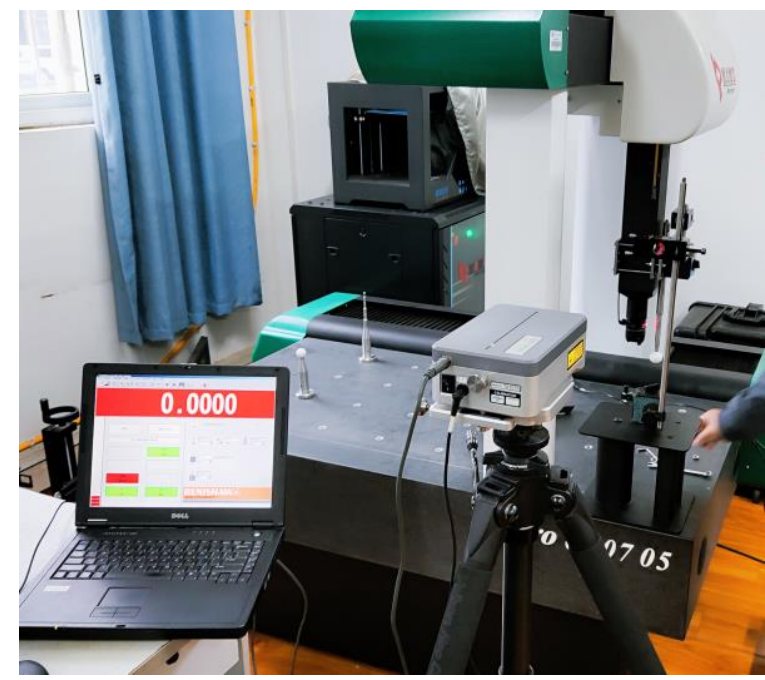

Fig. 13 The verification scene on the CMM with the aid of laser interferometer after calibration.

Tab. 6 Uncertainty of calibration for the normal vector $\boldsymbol{n}_{i}$ of the detection plane of sensors and the sensor position coordinates $S_{i_{-} 1}$. 


\begin{tabular}{cccc}
\hline $\boldsymbol{n}_{i}$ and $S_{i_{-} 1}$ & \multicolumn{3}{c}{ Standard uncertainty $u$} \\
\cline { 2 - 4 } & Senser 1 & Senser 2 & Senser 3 \\
\hline$a_{i}$ & 1.3622 & 1.9359 & 1.7610 \\
$b_{i}$ & 1.3035 & 1.9725 & 1.8797 \\
$c_{i}$ & 1.3549 & 1.9662 & 1.7736 \\
$x_{i_{-} 1}$ & 1.9864 & 1.3821 & 2.2175 \\
$y_{i_{-} 1}$ & 2.3450 & 1.9138 & 1.8652 \\
$z_{i_{-} 1}$ & 2.0659 & 1.4484 & 1.8412 \\
\hline
\end{tabular}
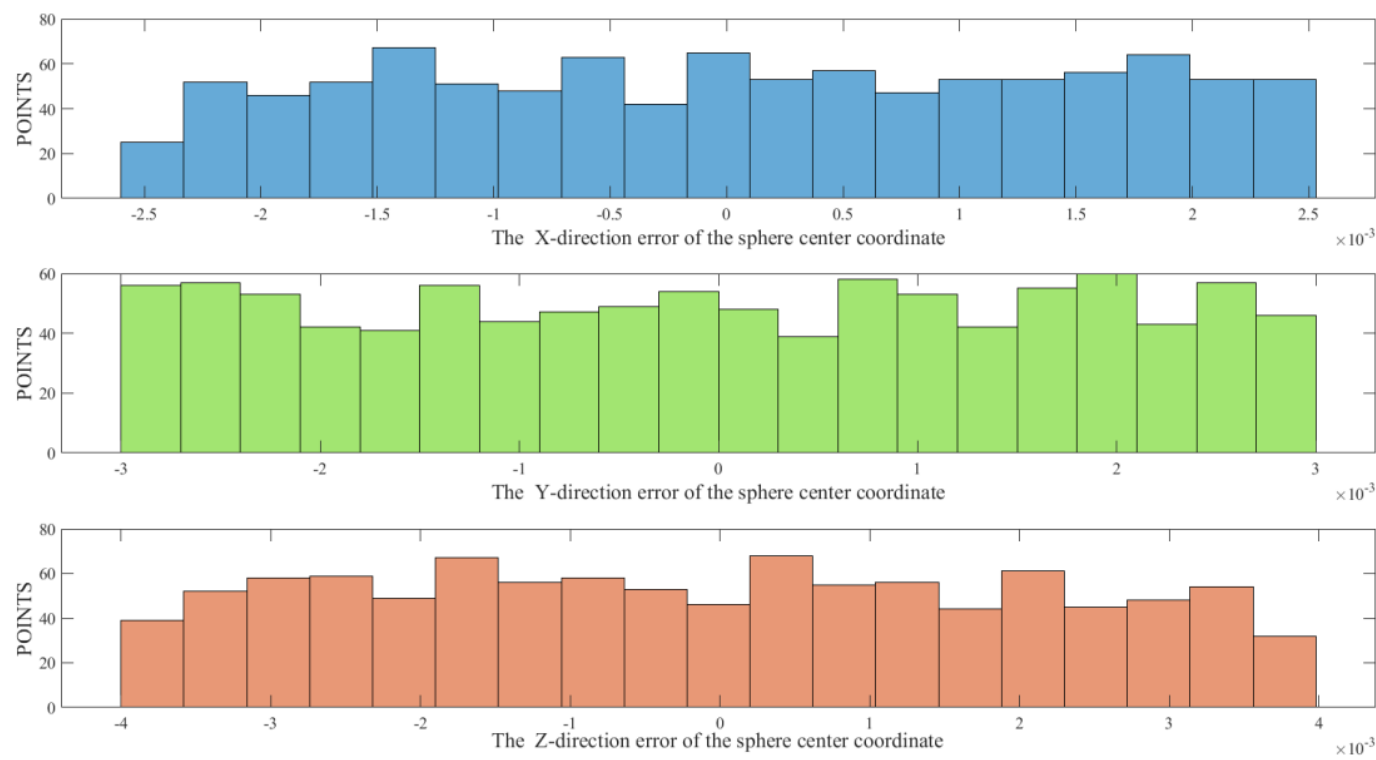

Fig. 14 Error graph of 1000 verification points in three directions after calibration.


Fig. 15 On-machine measurement scene of prototype on MIKRON machine tools.

\section{Conclusions}

In this paper, the novel hardware parameters configuration methods for the contact R-test are proposed to realize customization. The theoretical measurement model and the calibration model are established to evaluate the measurement accuracy of the measurement system. The constraint models for the measurement performance indexes of the system relative to hardware parameters are established, and to realize the optimal configuration of hardware parameters according to the constraint model through exhaustive search method and variable parameter method respectively. A prototype is developed according to the configuration methods, and a calibration experiment is carried out on a CMM. The verification results indicate that the optimized configuration methods 
of the hardware parameters can achieve the goal of customized configuration according to certain measurement requirements without precision and range waste. The hardware configuration methods proposed in this paper provide an important reference for the actual development and use of the R-test.

\section{Acknowledgements}

This work is supported by Sichuan Major Science and Technology Project of Intelligent Manufacturing and Robotics, China (2019ZDZX0021).

\section{The Declarations section}

a. Funding (information that explains whether and by whom the research was supported)

This work is supported by Sichuan Major Science and Technology Project of Intelligent Manufacturing and Robotics, China (2019ZDZX0021).

b. Conflicts of interest/Competing interests (include appropriate disclosures)

All authors certify that they have no affiliations with or involvement in any organization or entity with any financial interest or non-financial interest in the subject matter or materials discussed in this manuscript.

c. Availability of data and material (data transparency)

Not applicable.

d. Code availability (software application or custom code)

Not applicable.

e. Ethics approval (include appropriate approvals or waivers)

Not applicable.

f. Consent to participate (include appropriate statements)

All authors agreed to participate.

g. Consent for publication (include appropriate statements)

All authors agreed to publish.

h. Authors\&apos; contributions (optional: please review the submission guidelines from the journa 1 whether statements are mandatory)

All authors contributed to the study conception and design. The main content planning and preliminary plan determination are completed by Lei Jiang, the specific chapters and algorithm writing are completed by Yongbing Zhou, and the assistance in experiments and data analysis was 
provided by Guofu Ding and Yuexinkai Zhang. All authors commented on previous versions of the manuscript. All authors read and approved the final manuscript.

\section{References}

[1] Ding, Q, Wang, W, Du, L, Ding, J et al (2020) Dynamic performance test under complicated motion states for five-axis machine tools based on double ballbar[J]. Int J Adv Manuf Technol, 111(3-4):1-19. https://doi.org/10.1007/s00170-020-06101-3.

[2] Noriyuki Kato, Masaomi Tsutsumi, Ryuta Sato. (2013) Analysis of circular trajectory equivalent to cone-frustum milling in five-axis machining centers using motion simulator[J], Int. J. Mach.Tools Manuf. 64:1-11. https://doi.org/10.1016/j.ijmachtools.2012.07.013.

[3] Schwenke, H, Franke, M, Hannaford et al (2005) Error mapping of cmms and machine tools by a single tracking interferometer[J]. CIRP Annals - Manufacturing Technology, 54(1): 475-478. https://doi.org/10.1016/S0007-8506(07)60148-6.

[4] H. Schwenke, R. Schmitt, P. Jatzkowski, C. Warmann. (2009) On-the-fly calibration of linear and rotary axes of machine tools and CMMs using a tracking interferometer[J], CIRP Ann. Manuf. Technol. 58,477-480. https://doi.org/10.1016/j.cirp.2009.03.007.

[5] Wang, J, Cheng, C et al (2020) A novel approach to separate geometric error of the rotary axis of multi-axis machine tool using laser tracker[J]. International Journal of Precision Engineering and Manufacturing, 21(6):983-993. https://doi.org/10.1007/s12541-020-00329-5.

[6] Barman S, Sen R (2010). Enhancement of accuracy of multi-axis machine tools through error measurement and compensation of errors using laser interferometry technique[J]. Mapan, 25(2):79-87. https://doi.org/10.1007/s12647-010-0010-1.

[7] Weikert S (2004) R-Test, a New Device for Accuracy Measurements on Five Axis Machine Tools[J]. CIRP Ann Manuf Technol 53(1):429-432. https://doi.org/10.1016/S0007-8506(07)60732-X.

[8] ISO 230-7: test code for machine tools - part 7: geometric accuracy of axes of rotation, International Organization for Standardization(ISO), Geneva, Switzerland, 2015.

[9] Tsutsumi, M, Ihara, Y, Saito, A, Mishima et al (2008) A 18 standardization of testing methods for kinematic motion of five-axis machining centers : draft proposal for iso standard. The Proceedings of The Manufacturing \& Machine Tool Conference, 2008.7, 95-96. https://doi.org/ 10.1299/jsmemmt.2008.7.95.

[10] ISO/CD 10791-6. Test conditions for machining centers - part 6: Accuracy of feeds, speeds and interpolations[S]. Switzerland : ISO, 2009.

[11] Rotary analyzer user's manual, IBS Precision Engineering BV, Eindhoven, Netherlands, 2016.

[12] IBS Precision Engineering. Machine tool inspection and analyzer solutions [EB/OL]. http://ibspe.com/category/machine-tool-inspection-and-analyzer-solutions.htm.

[13] HMS (Head Measuring System), FIDIA S.p.A, San Mauro Torinese, Italy, 2018.

[14] Liu DW, Guo ZP, Song ZY (2013) An optimum structural design method of r-test sphere center measuring device[J]. J Mech Eng 49(23):161-166. https://doi.org/10.3901/JME.2013.23.161.

[15] Li LL, Du ZC (2013) Model analysis of a novel error measurement device of five-axis manufacturing center[J]. J Shanghai Jiao Tong Univ 47(11):1801-1806.

[16] Jiang, L, Peng, B, Ding, G, Qin, S et al (2020) Optimization method for systematically improving non-contact R-test accuracy[J]. Int J Adv Manuf Technol, 107(1) :1697-1711. https://doi.org/ 10.1007/s00170-020-04999-3. 
[17] Ibaraki, S, Yu, N, Otsubo, H, Sakai, Y, Morimoto, S et al (2015) R-test analysis software for error calibration of five-axis machine tools - application to a five-axis machine tool with two rotary axes on the tool side -[J]. Int J Adv Manuf Technol, 9(4), 387-395. https://doi.org/10.20965/ijat.2015.p0387.

[18] Li J, Xie FG, Li WD (2017) A geometric error identification method for the swiveling axes of five-axis machine tools by static R-test[J]. Int J Adv Manuf Technol 89(9-12):3393-3405. https://doi.org/ 10.1007/s00170-016-9199-7.

[19] Lei Z, Qingzhen B, Nuodi H, Yuhan W (2018) Dynamic accuracy evaluation for five-axis machine tools using $\mathrm{S}$ trajectory deviation based on R-test measurement[J]. Int J Mach Tools Manuf 125:20-33. https://doi.org/10.1016/j.ijmachtools.2017.11.003.

[20] Brecher, C, Behrens, J, Klatte, M, Lee, T. H et al (2018) Measurement and analysis of thermo-elastic deviation of five-axis machine tool using dynamic r-test. Procedia CIRP, 77:521-524. https://doi.org/10.1016/j.procir.2018.08.244.

[21] Hong C, Ibaraki S. (2013) Non-contact R-test with laser displacement sensors for error calibration o -f five-axis machine tools[J]. Precision Engineering, 37(1):159-171. https://doi.org/10.1016/j.precisionen g.2012.07.012.

[22] Bitar-Nehme, E, Mayer, J (2016) Thermal volumetric effects under axes cycling using an invar r-tes $\mathrm{t}$ device and reference length[J]. Int J Mach Tools Manuf, 105:14-22. https://doi.org/10.1016/j.ijmacht ools.2016.03.003.

[23] Weng, L, Zhang, D, Gao, W, Tian, Y et al (2020) Novel r-test measurement system to detect thermally induced volumetric accuracy in horizontal machine tools[J]. Measurement Science and Technology. https://doi.org/10.1088/1361-6501/ab9f43.

[24] Guo, Y, Song, B, X Tang, X Zhou et al (2020) A calibration method of non-contact r-test for erro $\mathrm{r}$ measurement of industrial robots[J]. Measurement, 173(1):108365. https://doi.org/ 10.1016/j.measure ment.2020.108365.

[25] ISO 10791-6: machine tools - test conditions for machining centres - part 6: accuracy of speeds and interpolations, International Organization for Standardization (ISO), Geneva, Switzerland, 2014.

[26] GBT27418-2017 Evaluation and Representation of Measurement Uncertainty[S]. Beijing: China Standard Press, 2017. 